\title{
Changes of the expression of Lewis blood group antigens in glycoproteins of renal cancer tissues
}

\author{
Małgorzata Borzym-Kluczyk ${ }^{\boxplus 凶}$ and Iwona Radziejewska² \\ 'Department of Pharmaceutical Biochemistry, ${ }^{2}$ Department of Medical Chemistry, Medical University of Bialystok, Białystok, Poland
}

Sialic acid and sialyl Lewis ${ }^{\mathrm{a} / \mathrm{x}}$ are found on N- and O-glycans of many human malignant cells. Carbohydrate antigens can be used as tumor markers, and an increase of their levels in cancer cells is associated with tumor progression. The aim of this study was to assess the level of some Lewis blood group antigens on glycoproteins in tumor (cancer tissue), intermediate zone (adjacent to tumor tissue), and normal renal cortex/medulla (uninvolved by tumor). The study was performed on tissues taken from 30 patients. Relative amounts of sugar structures of proteins with molecular masses above 30 $\mathrm{kDa}$ were determined by ELISA-like test with biotinylated lectins: MAA (Maackia amurensis), SNA (Sambucus nigra), and monoclonal antibodies anti-sialyl Lewis ${ }^{\mathrm{a} / \mathrm{x}}$. Higher expression of all examined structures was revealed in cancer tissues. Significant increases were observed for sialic acid linked a 2-3 in cancer tissues when compared to healthy ones and also among intermediate and healthy tissues. The sialic acid linked a 2-6 and sialyl Lewisx structures were significantly increased in cancerous cells when compared to normal and intermediate renal tissue. In case of sialyl Lewis a antigen, a significant difference was discovered between normal and intermediate tissue. Our results confirm that the examined Lewis antigens can be involved in tumor development. Their increase in cancer tissues can suggest their specific role in the process.

Key words: ELISA; glycosylation; renal cancer; sialyl Lewisa/x

Received: 18 February, 2013; 23 May, 2013; accepted: 05 June, 2013; available on-line: 14 June, 2013

\section{INTRODUCTION}

Tumor progression in an organism is connected with incorrect glycosylation of proteins. Changes in the synthesis and expression of sugar structures can occur. These changes play an important role not only in the initial stage of tumor progression, but also in subsequent stages related to tumor invasiveness and its ability of metastases formation (Spiro, 2002).

According to Gabius (Gabius et al., 2002), oligosaccharide chains may contain a vast quantity of biological information, which depends on sugar composition, their sequence, and spatial configuration. $\mathrm{N}$-and 0 -glycans differ in size, degree of branching, sugar composition, sequence, and also in charge. In glycoproteins, which appear physiologically, sialic acid is attached to the final galactose by a glycosidic linkage $\alpha 2-6$ and/or $\alpha 2-3$, as well as to $\mathrm{N}$-acetylgalactosamine by the $\alpha 2-6$ linkage (Kim et al., 1997). Sialic acid is a component of oligosaccharide determinants of sialyl Lewis type, which belong to the group of oligosaccharide antigens accompanying tumors (Kannagi, 2004). The Lewis group system includes an antigen or a group of antigens, which expression depends on one or more homologous genes, connected closely to one another, with no recombination process between them. Changes in the expression of group antigens of Lewis system accompany many physiological and pathological processes that occur in a human body. Lewis glycotypes are a heterogenic group of antigens with a common core (Galß1-3GlcNAc-type I or Galß1-4GlcNAc-type II), to which a particle of fucose is attached. These structures may occur in the sialylated form of Lewis ${ }^{a}\left(s L e{ }^{a}\right.$ ) and Lewis ${ }^{x}(s L e x)$, due to the attachment of sialic acid to a galactose particle by linkage a2-3 (Potapenko et al., 2010).

In cells that undergo cancerous transformation, one can observe a change in the expression of one or more oligosaccharide structures, such as NeuAc $\alpha 2-$ 3Gal; NeuAc $\alpha 2-6 \mathrm{Gal} / \mathrm{GalNAc}$; NeuAc $\alpha 2-3 \mathrm{Gal} \beta 1$ 4GlcNAc $\alpha$ Fuc $\alpha 1-4$ (sLe ${ }^{a}$, and NeuAc $\alpha 2-3$ Gal $\beta 1-$ 4GlcNAcFuc $\alpha 1-3$ (sLex) (Carvalho et al., 2010). There are two mechanisms responsible for changes in the expression. The first is based on an incomplete synthesis of oligosaccharides, which can be found on the surface of correct cells, such as 6-sulfo sialyl Lewis ${ }^{x}$ and disialo Lewisa. The other mechanism is the neosynthesis, which is based on raising of the glycosyltransferases expression (Kannagi, 2004). Fucosyltransferase Fuc-T III takes part in the biosynthesis of all Lewis type antigens. The synthesis of sialyl Lewis antigen happens as a result of the activity of ST3Gal-I, ST3Gal-II sialyltransferase, and most of all, ST3Gal-III, which catalyses the attachment of sialic acid to type I precursor chain. Sialyl Lewis antigen arises with the participation of ST3Gal-IV and ST3 Gal-VI, which catalyze the sialylation of type II precursor chain (Cazet et al., 2010).

The aim of our work was to assess differences in the glycosylation of proteins with molecular mass above 30 $\mathrm{kDa}$ in tumor (C), intermediate (I), and morphologically correct $(\mathrm{N})$ renal tissues. Expression of sialic acid, sialyl Lewis $^{a}$, sialyl Lewis ${ }^{\mathrm{x}}$ was estimated.

\section{MATERIAL AND METHODS}

The study was performed on renal tissues taken from 30 patients with clear cell renal cell carcinoma kidney cancer, hospitalized in the Department of Urology of the Medical University of Bialystok in Poland. Their diagno-

e-mail: gocha@umb.edu.pl

Abbreviations: BSA, bovine serum albumin; ELISA, enzyme-linked immunosorbent assay; Fuc-T, Fucosyltransferase; GalNAC, N-acetylgalactosamine; GIcNAc, N-acetylglucosamine; NeuAc, N-acetylneuraminic acid; sLex, sialyl Lewisx; sLea, sialyl Lewis ${ }^{a}$ 
ses were confirmed by histopathological assessment in the Department of Pathological Anatomy of the Medical University of Bialystok. The age of patients ranged from 51 to 76 years (mean age 63,15 females and 15 males). Kidneys were subjected to complete nephrectomy, and then sectioned in a way to obtain a cross-section plane passing through the tumor center and the long axis of the tumor. Tumor size, Fuhrman nuclear grade, and TNM stage are presented in Table 1. Tissue samples $(2 \times 2 \times 2 \mathrm{~cm})$ were taken from the tumor (C) intermediate zone, which was adjacent to tumor tissue (I), and normal $(\mathrm{N})$ renal cortex/medulla, which was untouched by tumor. Samples were rinsed with saline, dried with blotting paper and stored at $-70^{\circ} \mathrm{C}$.

Renal tissues were defrosted and immersed in $0.1 \mathrm{M}$ citric buffer, $\mathrm{pH} 4.3$, in order to prepare $10 \%$ (w/v) tissue suspensions. Then, tissues were homogenized in ULTRA-TURRAX T8 homogenizer for $2 \mathrm{~min}$. The obtained homogenate was centrifuged at $10000 \times g$, at $4^{\circ} \mathrm{C}$ for $20 \mathrm{~min}$. The supernatants were concentrated in Centriprep C30 concentrators (Amicon, Millipore, Bedford, MA, USA). Concentrated samples were applied on a Sephadex G-10 column and eluted with water. Collected $2 \mathrm{ml}$ fractions were assayed for absorbance at $280 \mathrm{~nm}$. The collected fractions were concentrated in Centriprep C30 (Amicon, Millipore, Bedford, MA, USA) concentrators, by repeated centrifugation at $1500 \times g$ for $60 \mathrm{~min}$, and then the supernatants were taken to further analyses. The concentration of protein in the samples was 40-60 mg protein $/ \mathrm{mL}$. The protein content was determined using bicinchoninic acid (Smith et al., 1985). Sugar structures of isolated proteins were analyzed using ELISA-like test with biotinylated lectins (Vector, Burlingame, USA) and monoclonal antibodies (Millipore, California, USA). For binding specificity of lectins and monoclonal antibodies see Table 2. Samples of renal tissue were diluted in the PBS buffer (Phosphate-Buffered Saline), and aliquots $(50 \mu \mathrm{L})$ were coated onto microtiter plates (NUNC F96; Maxisorp, Roskilde, Denmark) at room temperature overnight. The plates were washed 3 times $(100 \mu \mathrm{L})$ with PBS, pH 7.4, 0.05\% Tween (PBS$\mathrm{T}$; washing buffer) between all ensuing steps. Unbound sites were blocked with $100 \mu \mathrm{L}$ of $1 \%$ blocking reagent for ELISA (Roche Diagnostics, Mannheim, Germany) for $1 \mathrm{~h}$. The microtiter plates were incubated with 100 $\mu \mathrm{L}$ of biotinylated lectins $(1 \mathrm{~h})$, diluted to $0.5 \mu \mathrm{g} / \mathrm{mL}$ in PBS-T, 1\% bovine serum albumin, BSA (Sigma, St Luis, MO, USA). Lectin solutions were supplemented with metal cations: SNA with $0.1 \mathrm{mmol} / \mathrm{L} \mathrm{CaCl}_{2}$; $\mathrm{MAA}$ with $0.1 \mathrm{mmol} / \mathrm{L} \mathrm{CaCl}_{2}$ and $0.01 \mathrm{mmol} / \mathrm{L} \mathrm{MnCl}_{2}$. Plates were then incubated with $100 \mu \mathrm{L}$ of horseradish peroxidase avidin D (Vector, Burlingame, CA, USA) (1:2500) in PBS-T, 1\% BSA for $1 \mathrm{~h}$. Next, the plates were washed 4 times in PBS, and the colored reaction was developed by incubation with $100 \mathrm{~mL}$ of 2,2'-azino-bis(3-ethylbenzthiazoline-6-sulfonic acid) (ABTS)-liquid substrate for horseradish peroxidase (Sigma, St Luis, MO, USA). Spectrophotometric measurements were performed after 45 min at $405 \mathrm{~nm}$ using the Infinite $\mathrm{M}_{200}$ microplate reader (Tecan, Salzburg, Austria). For analysis with monoclonal antibodies (Millipore, California, USA) (diluted 1:500 in washing buffer, $1 \% \mathrm{BSA}$ ), the plates were coated with $100 \mu \mathrm{L}$ of antibody for $1 \mathrm{~h}$. Next, they were incubated with a secondary antibody, horseradish peroxidase-conjugated anti-mouse $\operatorname{IgG}$ (for anti-sialyl Lewis ${ }^{a}$ structure) or anti-mouse IgM (for anti-sialyl Lewis ${ }^{\mathrm{x}}$ structure) (Sigma, St Luis, MO, USA). The colored reaction was developed as mentioned before.

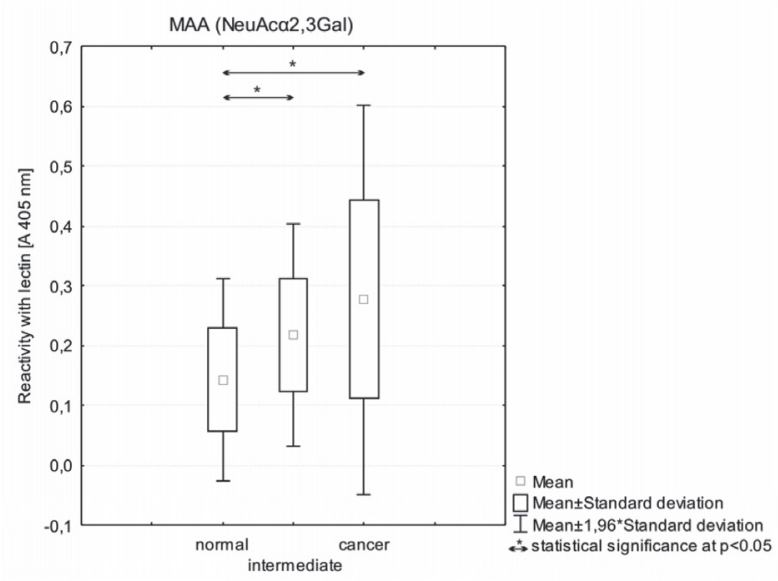

Figure 1. Comparison of reactivity with lectin MAA from Maackia amurensis in normal (N), intermediate (I), and cancer (C) renal tissue.

Statistical analysis. Statistical analyses were carried out using the Statistica 11 (StatSoft Cracow, Poland) software according to ANOVA and post hoc tests. Results are expressed as means \pm S.D. Statistical significance was defined as $p<0.05$.

The study was approved by the Institutional Ethical Committee and conducted according to the principles of the Declaration of Helsinki.

\section{RESULTS}

Glycoproteins of particular areas of renal tissues showed different reactivity with sialo specific lectins. To reveal the sialic acid linked by $\alpha 2-3$ to Gal, Maackia amurensis (MAA) and for $\alpha 2-6$ to Gal/GalNAc, Sambucus nigra (SNA) lectins were used. All the examined tissues showed higher amount of NeuAc $\alpha 2-6$ than in case of NeuAc $\alpha 2-3$ bindings. Both linkages showed significantly higher levels in cancer tissues when compared to healthy ones (Fig. 1, 2). Significant differences for the MAA lectin were observed for normal $(\mathrm{N})$ vs. intermediate tissue (I), and for normal $(\mathrm{N})$ vs. cancer tissue (C).

The expression of sialyl Lewis ${ }^{a}$ structure (NeuAc $\alpha 2$ 3Galß1-4 GlcNAc $\alpha 1-4 \mathrm{Fuc})$ was significantly higher than sialyl Lewis ${ }^{x}$ structure (NeuAc $\alpha 2-3$ Gal $\beta 1-4$ GlcNAc $\alpha 1-$

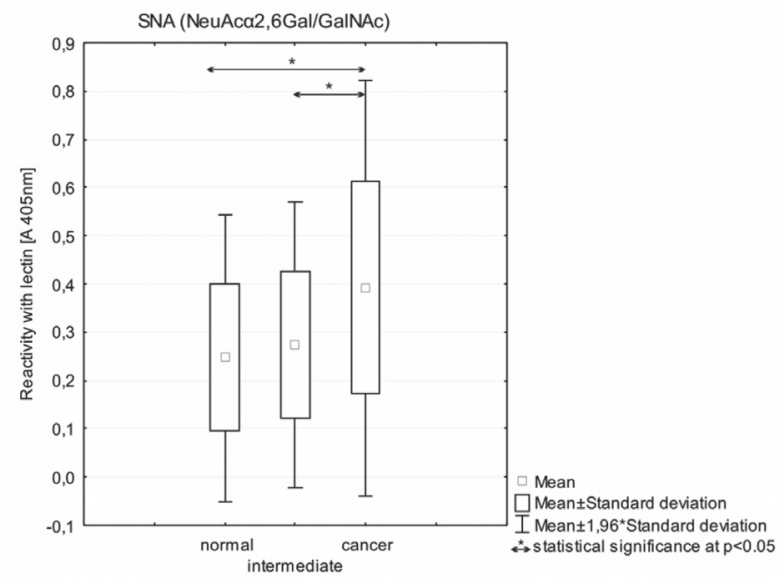

Figure 2. Comparison of reactivity with lectin SNA from Sambucus nigra in normal $(\mathrm{N})$, intermediate $(\mathrm{I})$, and cancer $(\mathrm{C})$ renal tissue. 


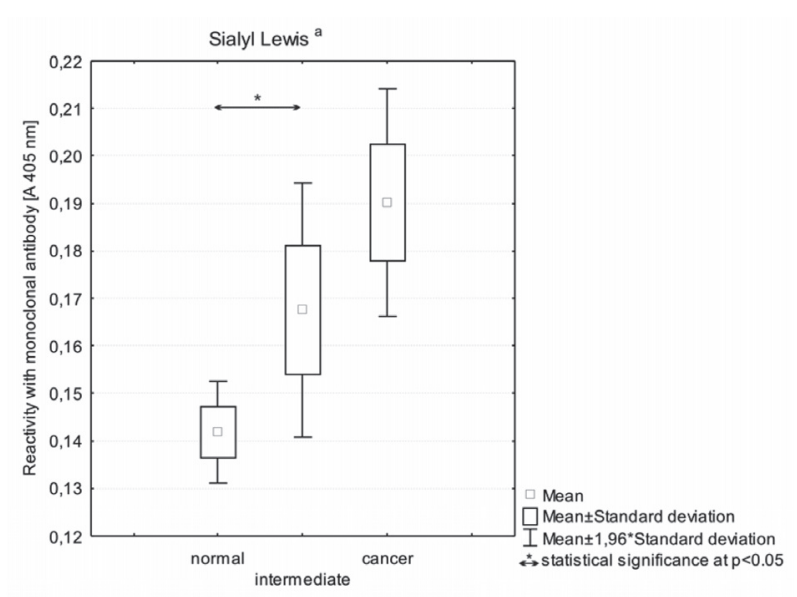

Figure 3. Comparison of reactivity with monoclonal antibody sialyl Lewis ${ }^{a}$ in normal (N), intermediate (I), and cancer (C) renal tissue.

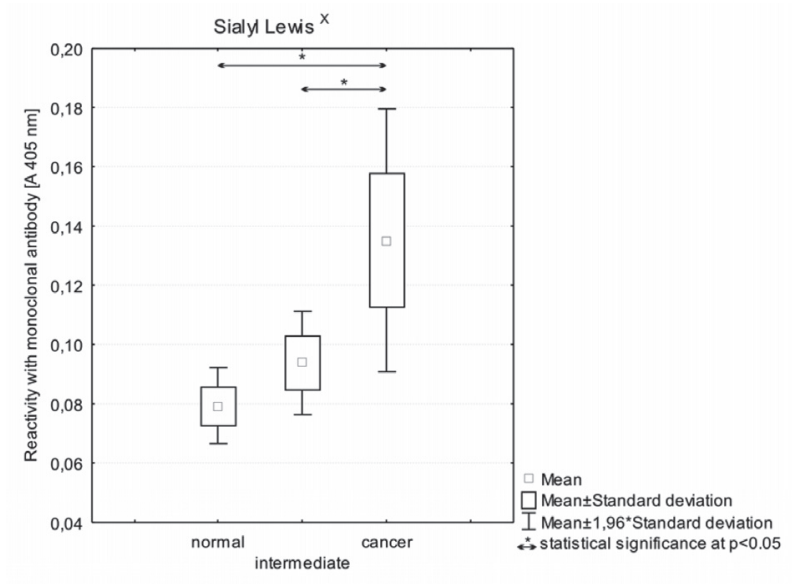

Figure 4. Comparison of reactivity with monoclonal antibody sialyl Lewis ${ }^{\mathrm{x}}$ in normal $(\mathrm{N})$, intermediate (I), and cancer (C) renal tissue.

3Fuc) (antigens were recognized by monoclonal antibodies). The expression of both linkages was significantly higher in cancer tissues (C) $\left(p<0.05\right.$ for sialyl Lewis ${ }^{a}$ and sialyl Lewis ${ }^{\mathrm{x}}$ ) when compared to healthy tissues $(\mathrm{N})$ (Fig. 3, 4).

We observed a significantly higher level of reactivity for the SNA lectin (Fig. 2) and monoclonal antibody anti sialyl Lewis ${ }^{\mathrm{x}}$ (Fig. 4) in proteins isolated from can-

Table 1. Number by size, Fuhrman nuclear grade, and TNM tumor stage.

\begin{tabular}{llllllllll}
\hline \multirow{2}{*}{ Lesion Size* and Mode of Presentation } & \multicolumn{3}{c}{ Fuhrman nuclear grade } & \multicolumn{4}{c}{ TNM tumor stage } \\
\cline { 2 - 11 } & G1 & G2 & G3 & G4 & T1 & T2 & T3 & T4 \\
\hline No.30 & 2 & 22 & 2 & 4 & 16 & 10 & 3 & 1 \\
\hline
\end{tabular}

*All tumors were $\leq 3 \mathrm{~cm}$

Table 2. Lectins and monoclonal antibodies and their binding specificity.

\begin{tabular}{ll}
\hline Lectins and monoclonal antibodies & Binding preference \\
\hline Maackia amurensis (MAA) & NeuAc $\alpha 2-3 G$ Gal \\
\hline Sambucus nigra (SNA) & NeuAc $\alpha 2-6 G a l / G a l N A C$ \\
\hline Mouse anti-sialyl Lewis × monoclonal antibody & NeuAc $\alpha 2-3 G$ Gal $\beta 1-4 G I C N A c \alpha 1-3 F u c$ \\
\hline Mouse anti-sialyl Lewis a monoclonal antibody & NeuAc $\alpha 2-3 G a l \beta 1-4 G I C N A c \alpha 1-4 F u c$ \\
\hline
\end{tabular}

cer tissues than those isolated from intermediate ones $(p<0.05)$.

\section{DISCUSSION}

Malignant transformation of tumor is associated with abnormal glycosylation resulting in the expression of altered carbohydrate determinants including sialyl Le and sialyl Le ${ }^{\mathrm{x}}$ antigens (Ugorski et al., 2002). Renal cell carcinoma (RCC) is a kidney cancer that originates from the lining of the proximal convoluted tubule. According to Cordon (Cordon-Cardo et al., 1989) the histogenesis of renal cell carcinoma in the proximal nephron is based on the expression of Lewis ${ }^{\mathrm{a}}$ and Lewis ${ }^{\mathrm{x}}$ antigens. It also shows: (a) an apparent deletion, down regulation or structural modification of $\mathrm{Le}^{\mathrm{x}}$ determinant in most of the metastatic tumors; (b) undetectable levels of $\mathrm{ABH}$ specificities in tumor cells of primary renal cell carcinoma; and (c) enhanced expression and/or neosynthesis of precursor type 1 structure and Lewis ${ }^{y}$ determinant in some renal cell carcinomas.

The results of our study confirm the majority of the above mentioned observations, revealing higher levels of some specific glycoforms in renal cancer tissue. In the process of fetal development, sialyl Lewis antigen is present in many tissues, such as bronchi, trachea, or larynx.

In adults, that antigen is present rather in small amounts in a healthy state. The increased expression of sialyl Lewis antigen is connected with metastasis ability of tumor cells of pancreas and colon and with a lowered survival rate of patients. As for patients with kidney cancer, we have demonstrated an increased expression of that structure. The overexpression of sialyl Lewis antigen is related to the increase of $\alpha 2-3$ sialyltransferase activity (Kannagi, 2004; Ugorski et al., 2002).

Sialic acid appearing on antigens characteristic for cancers may reveal masking effect by covering these antigens. In the process of biosynthesis, sialic acid often inhibits glucosyltransferase and blocks the further elongation of a sugar chain. However, its presence may also be required as a signal for other transferases ( $\mathrm{Li}$ et al., 2012). Sialyl Lewis ${ }^{\mathrm{x}}$ antigen can be found in some healthy cells of gallbladder epithelium, bronchioli epithelium, as well as in proximal renal tubules and granulocytes. Its increased expression was found in the cells of lung cancer, colon, prostate, urinary bladder, stomach, kidney and breast (Brockhausen, 2003). It was demonstrated that a large concentration of that structure determines a poor prognosis and a tendency of cancerous cells to spread onto lymph nodes. In the cells of kidney cancer examined by authors, an increased expression of sialyl Lewis $^{x}$ antigen was revealed, which is in accordance with our results. However, our study was performed on human tissue, not on cell lines. The overexpression of sialyl Lewis ${ }^{\mathrm{x}}$ antigen is connected with an increased level of mRNA for relevant genes (FUT3, FUT5, FUT6, FUT7) coding fucosyltransferases, which take part in sLe $^{\mathrm{x}}$ synthesis (Brockhausen, 2006; St Hill, 2011).

RCC is a highly metastatic type of cancer showing preferential blood-borne metastasis to lung as compared to liver or kidney or distant lymph nodes 
(Saito et al., 1991). A possible correlation of metastatic potential with sialyl Lewis ${ }^{x}$ and sialyl Lewis ${ }^{a}$ expression, the target of E- and P-selectin in tumor cells, is a major focus of recent studies (Nakamori et al., 1993). Selectins are integral membrane proteins, which differ in construction, location, and specifity. Their role is to direct leukocytes to an inflammated place. They are vital in the process of adhesion of leukocytes to endothelial tissue. Expression of sialyl Lewis ${ }^{\mathrm{x}}$ and sialyl Lewis ${ }^{\mathrm{a}}$ in primary tumors can be closely correlated with invasiveness (Matsushita et al., 1991), metastasis (Irimura et al., 1993), and inversely correlated to patient survival (Nakamori et al., 1993). However, according to Satoh (Satoh et al., 1996), the expression of sialyl Lewis ${ }^{\mathrm{x}}$ has no correlation with metastatic potential or degree of malignancy in the eight RCC lines tested. None of the cell lines showed E- or P-selectin-dependent adhesion. Recent results suggest the existence of yet-uncharacterized sialoadhesive receptors that recognize a specific structure - DSGG (disialosyl galactosylgloboside (NeuAc $\alpha 2-3 \mathrm{Gal} \beta 1-3$ (NeuAc $\alpha 2-6)$ GalNAc $\beta 1-3 G a l \alpha 1-4 G a l \beta 1-4 G l c \beta 1-1 c e r)$. This receptor could be the binding target in RCC metastasis to lung (Satoh et al., 1996).

Our results confirm that the examined specific sugar structures can be involved in tumor development. Although these antigens accumulate in tumor tissues of patients with Carcinoma clarocellulare, their functional roles have remained largely unknown.

We want to emphasize that our results should be treated as preliminary. These results need to be supplemented by the correlation with metastatic potential or degree of malignancy of renal cancer tissue.

\section{Acknowledgments}

We would like to express our great appreciation to Professor B. Darewicz, Head of the Department of Urology of the Medical University of Bialystok, for the provision of clinical material for the research.

\section{REFERENCES}

Brockhausen I (2003) Glycodynamics of mucin biosynthesis in gastrointestinal tumor cells. Adv Exp Med Biol 535: 163-188.

Brockhausen I (2006) Mucin-type O-glycans in human colon and breast cancer: glycodynamics and functions. EMBO Rep 7: 599-604.

Carvalho AS, Harduin-Lepers A, Magalhães A, Machado E, Mendes N, Costa LT, Matthiesen R, Almeida R, Costa J, Reis CA (2010) Dif- ferential expression of alpha-2,3-sialyltransferases and alpha-1,3/4fucosyltransferases regulates the levels of sialyl Lewis a and sialyl Lewis $\mathrm{x}$ in gastrointestinal carcinoma cells. Int J Biochem Cell Biol 42: 80-89.

Cazet A, Julien S, Bobowski M, Burchell J, Delannoy P (2010) Tumour-associated carbohydrate antigens in breast cancer. Breast Cancer Res 12: 204.

Cordon-Cardo C, Reuter VE, Finstad CL, Sheinfeld J, Lloyd KO, Fair WR, Melamed MR (1989) Blood group-related antigens in human kidney: modulation of Lewis determinants in renal cell carcinoma. Cancer Res 49: 212-218.

Gabius HJ, André S, Kaltner H, Siebert HC (2002) The sugar code: functional lectinomics. Biochim Biophys Acta 1572: 165-177.

Irimura T, Nakamori S, Matsushita Y, Taniuchi Y, Todoroki N, Tsuji T, Izumi Y, Kawamura Y, Hoff SD, Cleary KR et al. (1993) Colorectal cancer metastasis determined by carbohydrate-mediated cell adhesion: role of sialyl-LeX antigens. Semin Cancer Biol 4: 319-324.

Kannagi R (2004) Molecular mechanism for cancer-associated induction of sialyl Lewis X and sialyl Lewis A expression - the Warburg effect revisited. Glycoconj J 20: 353-364.

Kim YJ, Varki A (1997) Perspectives on the significance of altered glycosylation of glycoproteins in cancer. Glycoconj J 14: 569-576.

Li Y, Chen X (2012) Sialic acid metabolism and sialyltransferases: natural functions and applications. Appl Microbiol Biotechnol 94: 887-905.

Matsushita Y, Nakamori S, Seftor EA, Hendrix MJ, Irimura T (1991) Human colon carcinoma cells with increased invasive capacity obtained by selection for sialyl-dimeric LeX antigen. Exp Cell Res 196: 20-25.

Nakamori S, Kameyama M, Imaoka S, Furukawa H, Ishikawa O, Sasaki Y, Kabuto T, Iwanaga T, Matsushita Y, Irimura T (1993) Increased expression of sialyl Lewis ${ }^{\mathrm{x}}$ antigen correlates with poor survival in patients with colorectal carcinoma: clinicopathological and immunohistochemical study. Cancer Res 53: 3632-3637.

Potapenko IO, Haakensen VD, Lüders T, Helland A, Bukholm I, Sørlie T, Kristensen VN, Lingjaerde OC, Børresen-Dale AL (2010) Glycan gene expression signatures in normal and malignant breast tissue; possible role in diagnosis and progression. Mol Oncol 4: 98118.

Saito S, Orikasa S, Ohyama C, Satoh M, Fukushi Y (1991) Changes in glycolipids in human renal-cell carcinoma and their clinical significance. Int J Cancer 49: 329-334.

Satoh M, Handa K, Saito S, Tokuyama S, Ito A, Miyao N, Orikasa S, Hakomori S (1996) Disialosyl galactosylgloboside as an adhesion molecule expressed on renal cell carcinoma and its relationship to metastatic potential. Cancer Res 56: 1932-1938.

Smith PK, Krohn RI, Hermanson GT, Makia AK, Gartner FH, Provwnzano MD, Fujimot EK, Goeke NM, Olson BJ, Kleuk DC (1985) Measurement of protein using bicinchoninic acid. Anal Biochem 150: 1530-1539.

Spiro RG (2002) Protein glycosylation: nature, distribution, enzymatic formation, and disease implications of glycopeptide bonds. Glycobiology 12: 43R-56R.

St Hill CA (2011) Interactions between endothelial selectins and cancer cells regulate metastasis. Front Biosci 17: 3233-3251.

Ugorski M, Laskowska A (2002) Sialyl Lewis(a): a tumor-associated carbohydrate antigen involved in adhesion and metastatic potential of cancer cells. Acta Biochim Pol 49: 303-311. 